\title{
Novel multilayer designs for future hard x-ray missions
}

F. E. Christensen, C. P. Jensen, K. K. Madsen, M. J. Pivovaroff, $\mathrm{H}$. Chen, et al.

F. E. Christensen, C. P. Jensen, K. K. Madsen, M. J. Pivovaroff, H. Chen, A. Dariel, P. Høghøj, "Novel multilayer designs for future hard x-ray missions," Proc. SPIE 6266, Space Telescopes and Instrumentation II: Ultraviolet to Gamma Ray, 626611 (13 June 2006); doi: 10.1117/12.673181

SPIE Event: SPIE Astronomical Telescopes + Instrumentation, 2006, Orlando, Florida, United States 


\title{
Novel multilayer designs for future hard X-ray missions
}

\author{
F. E. Christensen ${ }^{\text {a }}$, C. P. Jensen ${ }^{\mathrm{a}}$, K. K. Madsen ${ }^{\mathrm{a}}$, M. J. Pivovaroff ${ }^{\mathrm{b}}$, H. Chen ${ }^{\mathrm{c}}$, A. Dariel ${ }^{\mathrm{d}}$, P. Høghøj ${ }^{\mathrm{d}}$ \\ ${ }^{a}$ Danish National Space Center, 2100 Copenhagen, Denmark \\ ${ }^{\mathrm{b}}$ Lawrence Livermore National Laboratory, Livermore CA 94550, USA \\ ${ }^{\mathrm{c} S}$ Space Radiation Laboratory, California Institute of Technology, Pasadena CA 91125, USA \\ ${ }^{d}$ XENOCS, F-38360 Sassenage, France
}

\begin{abstract}
Current multilayer designs for $10-80 \mathrm{keV}$ hard X-ray telescope missions have focused primarily on the proven properties of $\mathrm{W}$ and $\mathrm{Pt}$ based multilayer coatings. Recently a number of new material combinations and coating capabilities have emerged which allows for more elaborate designs that can further extend the energy band of current mission designs as well as avoid some of the unwanted absorption edge effects in the effective area near potentially important line emission energies. These new design possibilities are investigated for current hard X-ray mission designs. The new material combinations to be considered are recently proven capabilities of enhanced NiV/C coatings and $\mathrm{NiV} / \mathrm{SiC}$ coatings in conjuction with the well-established $\mathrm{W}$ based coatings.
\end{abstract}

Keywords: X-ray multilayers, Hard X-ray Astronomy, X-ray optics

\section{INTRODUCTION}

In addition to the current hard X-ray balloon programs such as HEFT [1] and InFocus [2] a number of future hard X-ray satellite missions are based on the use of depth graded multilayer coatings to enable focusing at energies significantly above $10 \mathrm{keV}$. The depth grading acts as a broad band reflector with the period (or so called d-spacing) varying continuously through the stack with the hardest X-rays being reflecting in the bottom of the stack. These missions include NuSTAR [3], HXT on Con-X [4], NeXT [5], the hard X-ray extension of the XEUS mission concept [6] and possibly a hard X-ray focusing telescope for the GRI mission concept[7]. All of these missions are aiming at achieving significant effective area above $40 \mathrm{keV}$ thus covering the energy band needed to study the hard X-ray background thought to originate from obscured AGN's - one of the primary science drivers for focusing missions in this energy band. Another key objective for a focusing mission in the hard X-ray band is the study of line emission from radioactive ${ }^{44} \mathrm{Ti}$ thought to originate during supernova explosions near the mass cut where matter is either ejected into the surroundings or falls back on the compact object. This line emission is at $67.9 \mathrm{keV}$ and $78.4 \mathrm{keV}[8]$.

Current hard X-ray telescope designs are based on the use of either $\mathrm{W}$ and/or Pt as the reflecting layer. These have absorption edges at $69.5 \mathrm{keV}$ and $78.4 \mathrm{keV}$ respectively and the resulting effective area curves display sharp drops above these energies. These are uncomfortably close to the line emission energies and it becomes difficult to determine the continuum around the lines using these designs. Finally there is a desire to extend the energy band to even higher energies as important line emission occurs in the $100-200 \mathrm{keV}$ band foremost from ${ }^{56} \mathrm{Ni}$ at $158 \mathrm{keV}$ [8], [9] but also ${ }^{57} \mathrm{Co}$ at $122 \mathrm{keV}$ and $136 \mathrm{keV}$ [8]. For these reasons it would be desirable to develop new coating designs which allows for smooth throughput up to and well above $100 \mathrm{keV}$. The development of novel material combinations and new coating capabilities over the past few years have paved the way for new design possibilities. A simple solution to avoiding the absorption edge cut offs is to use a reflecting layer which does not have an absorption edge at energies above $20 \mathrm{keV}$. Ni has long been a good candidate for this and we have recently used the nonmagnetic NiV alloy in combination with SiC as the spacer material [10] in our planar magnetron sputtering facility at the Danish National Space Center (DNSC). Likewise enhanced NiV/C coatings have been developed by the XENOCS company in Grenoble using their magnetron sputtering facility [6]. Another novel development has been the realization that by combining two material combinations in the depth graded coating one can avoid the undesirable large drop in effective area at the $\mathrm{k}$ absorption edges otherwise inherent if using only $\mathrm{W}$ or $\mathrm{Pt}$ as the reflecting layer. An obvious possibility is to let proven small d-spacing $\mathrm{W} / \mathrm{SiC}$ [11]

Space Telescopes and Instrumentation II: Ultraviolet to Gamma Ray, edited by

Martin J. L. Turner, Günther Hasinger, Proc. of SPIE Vol. 6266, 626611, (2006)

0277-786X/06/\$15 - doi: 10.1117/12.673181

Proc. of SPIE Vol. 6266 626611-1 
reflect the hardest X-ray energies and then on top of this use a Ni-based coating to reflect the lower energies. This type of dual material combination coating has recently been developed and demonstrated in our production coating mode at DNSC [10]. Finally in order to make realistic designs extending up to and above $100 \mathrm{keV}$ it is necessary to use measured optical constants in this energy range as the effect of Compton scattering make tabulated optical constants uncertain for energies above $100 \mathrm{keV}$. These have recently been measured [12] and have been used for the designs presented in this paper.

The paper is organized as follows. Section 2 describes the design baseline using various options for the realization of HXT on Con-X as a baseline. Section 3 shortly reviews the properties of the material combinations we are using and the limitations we impose based on this. After that a presentation of the results of the design optimizations is given in Section 4, and finally a conclusion is given in Section 5 .

\section{DESIGN BASELINE}

We have chosen to use various options for the realization of the HXT on Con-X as a specific example of how these new design possibilities may be applied to a future satellite mission. There is an ongoing effort to optimize the focal length of the Con-X telescope mission, with the currently favored range spanning from 10 to $15 \mathrm{~m}$. We have therefore chosen to look at 3 specific cases for the HXT on Con-X: a $10 \mathrm{~m}$, a $12.5 \mathrm{~m}$ and a $15 \mathrm{~m}$ focal length option with the number of HXT modules and satellites scaling as listed in Table 1 .

Table 1. Number of modules versus focal length.

\begin{tabular}{ccc}
\hline \hline Focal length, [m] & Number of satellites & Total number of modules \\
\hline 10.0 & 4 & 12 \\
12.5 & 3 & 9 \\
15.0 & 2 & 6 \\
\hline
\end{tabular}

For this study we are employing the conical approximation to the Wolter I design using the segmented thin glass approach for the HXT and we use this to evaluate the mass of the telescopes. Table 2 lists the baseline parameters for the telescope design. They are deduced from design experience from the NuSTAR hard X-ray mission and assuming appropriate performance enhancements as made possible by the new coating designs and current development work on the segmented glass substrates [13]. 
Table 2. Baseline telescope parameters

\begin{tabular}{lr}
\hline \hline Minimum radius & $5.9 \mathrm{~cm}$ \\
Maximum radius, $f=\{10.0,12.5,15.0 \mathrm{~m}\}$ & $\{17.5,21.9,26.3\} \mathrm{cm}$ \\
Maximum on-axis graze angle & $0.25 \mathrm{deg}$ \\
Glass thickness & $0.2 \mathrm{~mm}$ \\
Glass density & $2.51 \mathrm{~g} / \mathrm{cm}^{3}$ \\
Length of each conical section & $300 \mathrm{~mm}$ \\
Spacing between upper and lower conical sections & $4 \mathrm{~mm}$ \\
Additional spacing between mirror shells & $0.17 \mathrm{~mm}$ \\
Assumed HPD, on-axis & $20 \mathrm{arcsec}$ \\
Maximum number of bilayers & 600 \\
Obscuration due to structure, spider, etc & $20 \%$ \\
\hline
\end{tabular}

Using these parameters the number of shells per telescope follow directly and thus the total glass mass can be calculated. These numbers are given in Table 3.

Table 3. Number of shells per module and total glass mass

\begin{tabular}{ccccc}
\hline \hline $\begin{array}{c}\text { Focal } \\
\text { length }\end{array}$ & $\begin{array}{c}\text { Number } \\
\text { of } \\
\text { Modules }\end{array}$ & $\begin{array}{c}\text { Number of } \\
\text { shells } \\
\text { per module }\end{array}$ & $\begin{array}{c}\text { Mass of glass } \\
\text { shells } \\
\text { per module } \\
{[\mathbf{k g}]}\end{array}$ & $\begin{array}{c}\text { Total mass } \\
\text { of glass shells } \\
{[\mathbf{k g}]}\end{array}$ \\
\hline 10.0 & 12 & 96 & 14.5 & 174 \\
12.5 & 9 & 139 & 24.5 & 220.5 \\
15.0 & 6 & 184 & 37.0 & 222.0 \\
\hline
\end{tabular}

We are making two separate designs. The first design is based solely on the use of the novel NiV/SiC coatings [10] using the measured performance regarding the minimum d-spacing and interfacial roughness as summarized in the next section. The second design uses a combination of $\mathrm{W} / \mathrm{SiC}$ in the bottom of the stack and NiV/SiC on top. Again we are using the measured performance of $\mathrm{W} / \mathrm{SiC}$ as presented in the next section. We are optimizing the coating design in 20 separate mirror groups logarithmically distributed throughout the on-axis graze angle range for the pure $\mathrm{NiV} / \mathrm{SiC}$ design . For each mirror group we are assigning an energy band by careful selection of the minimum $d$-spacing, $d_{\min }$ and maximum $d$-spacing, $d_{\max }$, for that mirror group and always within the limits set by the measured performance as given in the next section. 
The optimization for the $\mathrm{NiV} / \mathrm{SiC}$ case alone follow the method outlined in Peter Mao et al [14]. This uses a Figure Of Merit (FOM) which is an angular and energy weighted effective area. It assumes that the depth grading is a power law where the $\mathrm{i}^{\text {th }}$ bilayer thickness $\mathrm{d}(\mathrm{i})$ is given by :

$$
d(i)=\frac{a}{(i-b)^{c}}
$$

a,b.c are constants and $\mathrm{i}$ is the $\mathrm{i}^{\text {th }}$ bilayer ranging from 1 to $\mathrm{N}$ with $\mathrm{i}=\mathrm{N}$ being the bilayer next to the substrate. After selection of $d_{\min }$ and $d_{\max }$ for a given mirror group the parameters of the power law is determined by maximizing the FOM by running through a 3-dimensional parameter space of number of bilayers, $\mathrm{N}$, the power-law index $\mathrm{c}$ and the ratio between the thickness of the heavy element and the d-spacing, $\Gamma$. This determines uniquely the constants $\mathrm{a}, \mathrm{b}$ and $\mathrm{c}$.

The optimization of the combined $\mathrm{W} / \mathrm{SiC}$ and $\mathrm{NiV} / \mathrm{SiC}$ case is done in 11 mirror groups and entails a larger parameter space and we have chosen to use the On-axis effective area as the FOM for each mirror group for this case. Also we have chosen for each of the two material combinations to use a power law design. The parameters of the power law is determined by running through a multi parameter space of the two power-law indices, the two $\Gamma$ 's, the number of periods for each material combination and the d-spacing at which one shifts from one material combination to the next. The absolute $\mathrm{d}_{\min }$ and $\mathrm{d}_{\max }$ is, as noted above, set beforehand by selecting the energy band for each mirror group.

Together with the data and considerations regarding the performance of the material combinations given in the next section, the above is a complete set of prescriptions for doing the optimizations and calculating the resulting effective area and sensitivity for each of the 3 focal length cases.

\section{PROPERTIES OF NIV/SIC AND W/SIC MATERIAL COMBINATIONS}

The material combinations of interest to this design study as well as several other potential candidates have been investigated in detail in terms of their interfacial roughness versus the d-spacing, the minimum attainable d-spacing, their stress and the long term stability as well as resistance to proton irradiation. The status of these properties for a number of material combinations are summarized in the paper by Jensen et al [10] and Lumb et al [6]. In terms of the usefulness in a depth graded design for a specific multilayer material combination the essential parameters of interest are the interfacial roughness/interdiffusion and how small a d-spacing can be obtained. The reduction of the amplitude reflectivity at each interface due to roughness/interdiffusion is given by the exponential factor:

$$
\exp \left[-\left(\frac{2 \pi \sigma}{d}\right)^{2}\right]
$$

Where $\sigma$ is the Gaussian width of the roughness/interdiffusion at the interface. Thus $\sigma / d$ is the critical parameter of interest. From previous experience optimizing grazing incidence, hard X-ray, two reflection geometries such as the conical approximation to the Wolter I geometry we have generally found that $\sigma / d$ should be no larger than approximately $1 / 6$. At values larger than this it is no longer an optimal use of the material combination. Figure 1 below shows the experimentally determined values of $\sigma / \mathrm{d}$ for the material combinations relevant to this study, namely NiV/SiC and $\mathrm{W} / \mathrm{SiC}$. 

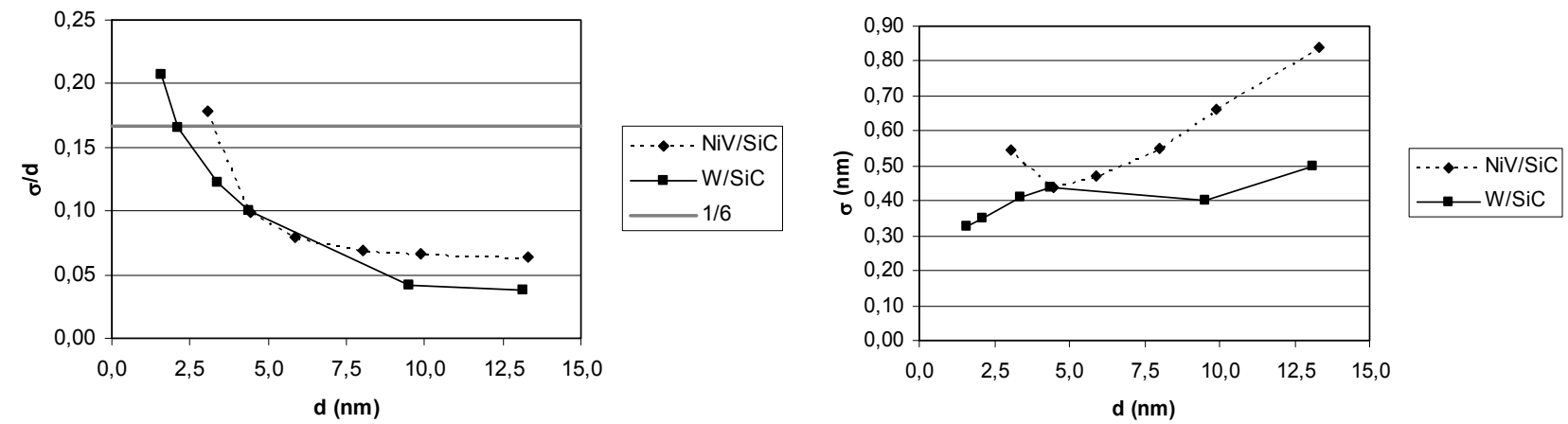

Figure 1. The measured $\sigma / \mathrm{d}$ (left) and $\sigma($ right) values for $\mathrm{NiV} / \mathrm{SiC}$ and $\mathrm{W} / \mathrm{SiC}$ material combinations. The thick line in the $\sigma / d$ curve corresponds to a $\sigma / d$ value of $1 / 6$ and is the value one needs to stay below for graded $d$-spacings designs of the type described in this paper.

From this figure it is evident that $\sigma / \mathrm{d}$ is smaller than $1 / 6$ for $\mathrm{NiV} / \mathrm{SiC}$ for $\mathrm{d}$-spacings above ca $35 \AA$ and likewise for $\mathrm{W} / \mathrm{SiC} \sigma / \mathrm{d}$ is smaller than $1 / 6$ for $\mathrm{d}$-spacings above ca $18 \AA$. This thus sets the limits for the smallest $\mathrm{d}$-spacings to be used for each material combination and the figure also gives the interface widths to be used for the optimization and we have chosen $5.5 \AA$ for $\mathrm{NiV} / \mathrm{SiC}$ and $3.5 \AA \mathrm{W} / \mathrm{SiC}$ as this is what they are at the smallest and most critical d-spacings. It is worth noting that these values are experimentally determined at the values of $\Gamma$ which are optimum for these designs.

\section{RESULTS OF OPTIMIZATIONS}

The results of the optimization for the $\mathrm{NiV} / \mathrm{SiC}$ alone is given in Table 4. This lists the 20 mirror groups, the energy band each mirror group is optimized over - which relates directly to the listed $d_{\min }$ and $d_{\max }$ for each group. The resulting parameters of the power law design is given by the c-value, the $\Gamma$ value and the number of bilayers. 
Table 4. Result of optimization for the NiV/SiC case alone

\begin{tabular}{cccccccc}
\hline $\begin{array}{c}\text { Mirror } \\
\text { group }\end{array}$ & $\begin{array}{c}\text { Angular range } \\
{[\mathbf{m r a d}]}\end{array}$ & $\begin{array}{c}\text { Energy band } \\
{[\mathbf{k e V}]}\end{array}$ & $\begin{array}{c}\mathbf{d}_{\min } \\
{[\mathbf{\AA}]}\end{array}$ & $\begin{array}{c}\mathbf{d}_{\max } \\
{[\AA \mathbf{\AA}]}\end{array}$ & $\mathbf{N}$ & $\mathbf{c}$ & $\boldsymbol{\Gamma}$ \\
\hline 1 & $1.200-1.280$ & $20.0-138.4$ & 35.0 & 516.6 & 566 & 0.240 & 0.513 \\
2 & $1.280-1.365$ & $20.0-129.8$ & 35.0 & 484.4 & 566 & 0.235 & 0.500 \\
3 & $1.365-1.455$ & $20.0-121.7$ & 35.0 & 454.2 & 566 & 0.235 & 0.500 \\
4 & $1.455-1.552$ & $20.0-114.1$ & 35.0 & 425.9 & 566 & 0.235 & 0.500 \\
5 & $1.552-1.655$ & $20.0-107.0$ & 35.0 & 399.4 & 566 & 0.232 & 0.470 \\
6 & $1.655-1.765$ & $20.0-100.3$ & 35.0 & 374.5 & 566 & 0.232 & 0.470 \\
7 & $1.765-1.882$ & $20.0-94.1$ & 35.0 & 351.2 & 566 & 0.232 & 0.470 \\
8 & $1.882-2.008$ & $20.0-88.2$ & 35.0 & 329.3 & 566 & 0.223 & 0.481 \\
9 & $2.008-2.141$ & $20.0-82.7$ & 35.0 & 308.8 & 566 & 0.235 & 0.500 \\
10 & $2.141-2.283$ & $20.0-77.6$ & 35.0 & 289.6 & 566 & 0.223 & 0.463 \\
11 & $2.283-2.435$ & $20.0-72.7$ & 35.0 & 271.5 & 566 & 0.215 & 0.480 \\
12 & $2.435-2.597$ & $20.0-68.2$ & 35.0 & 254.6 & 566 & 0.223 & 0.456 \\
13 & $2.597-2.769$ & $20.0-64.0$ & 35.0 & 238.7 & 566 & 0.217 & 0.463 \\
14 & $2.769-2.953$ & $20.0-60.0$ & 35.0 & 223.9 & 566 & 0.210 & 0.438 \\
15 & $2.953-3.149$ & $20.0-56.2$ & 35.0 & 209.9 & 453 & 0.221 & 0.465 \\
16 & $3.149-3.359$ & $20.0-52.7$ & 35.0 & 196.8 & 566 & 0.207 & 0.445 \\
17 & $3.359-3.582$ & $20.0-49.5$ & 35.0 & 184.6 & 453 & 0.210 & 0.437 \\
18 & $3.582-3.820$ & $20.0-46.4$ & 35.0 & 173.1 & 453 & 0.209 & 0.417 \\
19 & $3.820-4.073$ & $20.0-43.5$ & 35.0 & 162.3 & 566 & 0.184 & 0.399 \\
20 & $4.073-4.344$ & $20.0-40.8$ & 35.0 & 152.2 & 566 & 0.179 & 0.388 \\
\hline
\end{tabular}

The results for the optimization for the combined $\mathrm{NiV} / \mathrm{SiC}$ and $\mathrm{W} / \mathrm{SiC}$ are given in table 5 and 6 . Note that the number of mirror groups has been reduced, compared to the optimization performed solely for the $\mathrm{NiV} / \mathrm{SiC}$ design. Also note that we have chosen to use only $\mathrm{W} / \mathrm{SiC}$ for the three outermost mirror groups (9-11). This will leave a small drop at the W k-absorption edge but will increase the area slightly for energies below $70 \mathrm{keV}$ as opposed to making the effective area curve completely smooth by having all mirror groups use the dual material combination stack.

Table 5 Result of optimization for the combined NiV/SiC and W/SiC case: E-band, $\mathrm{d}_{\min }$ 's and $\mathrm{d}_{\max }$ 's

\begin{tabular}{|c|c|c|c|c|c|c|c|}
\hline \multirow[b]{2}{*}{$\begin{array}{l}\text { Mirror } \\
\text { group }\end{array}$} & \multirow[b]{2}{*}{$\begin{array}{c}\text { Angular range } \\
\text { [mrad] }\end{array}$} & \multirow[b]{2}{*}{$\begin{array}{c}\text { Material } \\
\text { combinations }\end{array}$} & \multirow[b]{2}{*}{$\begin{array}{c}\text { Energy band } \\
{[\mathrm{keV}]}\end{array}$} & \multicolumn{2}{|c|}{$\mathrm{NiV/SiC}$} & \multicolumn{2}{|c|}{$\underline{\mathrm{W} / \mathrm{SiC}}$} \\
\hline & & & & $\begin{array}{c}d_{\max } \\
{[\AA]}\end{array}$ & $\begin{array}{l}\mathbf{d}_{\min } \\
{[\AA]}\end{array}$ & $\begin{array}{c}\mathbf{d}_{\max } \\
{[\AA]}\end{array}$ & $\begin{array}{c}\overline{\mathbf{d}}_{\min } \\
{[\AA]}\end{array}$ \\
\hline 1 & $1.20-1.28$ & Both & $60.0-180$ & 400 & 50.0 & 52.0 & 32.0 \\
\hline 2 & $1.28-1.39$ & Both & $60.0-170$ & 400 & 50.0 & 52.0 & 30.0 \\
\hline 3 & $1.39-1.50$ & Both & $60.0-150$ & 400 & 45.0 & 47.0 & 30.0 \\
\hline 4 & $1.50-1.64$ & Both & $50.0-140$ & 400 & 38.0 & 40.0 & 27.5 \\
\hline 5 & $1.64-1.90$ & Both & $50.0-125$ & 400 & 35.0 & 37.0 & 25.0 \\
\hline 6 & $1.90-2.20$ & Both & $40.0-110$ & 400 & 35.0 & 37.0 & 25.0 \\
\hline 7 & $2.20-2.55$ & Both & $20.0-90.0$ & 250 & 35.0 & 37.0 & 25.0 \\
\hline 8 & $2.55-2.95$ & Both & $20.0-75.0$ & 250 & 35.0 & 37.0 & 25.0 \\
\hline 9 & $2.95-3.40$ & $\mathrm{~W} / \mathrm{SiC}$ & $20.0-70.0$ & $\mathrm{~N} / \mathrm{A}$ & $\mathrm{N} / \mathrm{A}$ & 215 & 25.0 \\
\hline 10 & $3.40-3.90$ & $\mathrm{~W} / \mathrm{SiC}$ & $20.0-70.0$ & $\mathrm{~N} / \mathrm{A}$ & $\mathrm{N} / \mathrm{A}$ & 160 & 23.0 \\
\hline 11 & $3.90-4.40$ & $\mathrm{~W} / \mathrm{SiC}$ & $20.0-70.0$ & $\mathrm{~N} / \mathrm{A}$ & $\mathrm{N} / \mathrm{A}$ & 140 & 20.0 \\
\hline
\end{tabular}


Table 6. Result of optimization for the combined NiV/SiC and W/SiC case: N's, c's, and $\Gamma$ 's

\begin{tabular}{ccccccc}
\hline \hline & \multicolumn{3}{c}{ NiV/SiC } & \multicolumn{3}{c}{ W/SiC } \\
Mirror group & $\mathbf{N}$ & $\mathbf{c}$ & $\Gamma$ & $\mathbf{N}$ & $\mathbf{c}$ & $\Gamma$ \\
\hline 1 & 110 & 0.22 & 0.47 & 90 & 0.26 & 0.47 \\
2 & 150 & 0.18 & 0.55 & 130 & 0.27 & 0.48 \\
3 & 205 & 0.18 & 0.50 & 140 & 0.25 & 0.48 \\
4 & 400 & 0.22 & 0.50 & 200 & 0.33 & 0.49 \\
5 & 430 & 0.21 & 0.49 & 170 & 0.29 & 0.47 \\
6 & 430 & 0.22 & 0.50 & 170 & 0.29 & 0.47 \\
7 & 460 & 0.235 & 0.52 & 140 & 0.330 & 0.44 \\
8 & 380 & 0.24 & 0.46 & 220 & 0.32 & 0.46 \\
9 & N/A & N/A & N/A & 330 & 0.225 & 0.38 \\
10 & N/A & N/A & N/A & 540 & 0.213 & 0.35 \\
11 & N/A & N/A & N/A & 590 & 0.235 & 0.36 \\
\hline
\end{tabular}

\subsection{Effective areas}

\subsubsection{NiV/SiC multilayers}

Figure 2 shows the total on-axis area for the full complement of modules for all three focal lengths of the NiV/SiC design. The $15.0 \mathrm{~m}$ focal design has significantly more area at higher energies, although the $12.5 \mathrm{~m}$ design has the largest area at the lowest energies. Table 7 lists the on-axis area at selected energies across the entire energy band.

Table 7: On-axis effective energy for the $\mathrm{NiV} / \mathrm{SiC}$ alone multilayer designs

\begin{tabular}{cccc}
\hline \hline & \multicolumn{3}{c}{ Effective Area [cm $\left.\mathrm{cm}^{2}\right]$} \\
Energy [keV] & $\mathrm{f}=10.0 \mathrm{~m}$ & $\mathrm{f}=12.5 \mathrm{~m}$ & $\mathrm{f}=15.0 \mathrm{~m}$ \\
\hline 10.0 & 4964 & 6014 & 5896 \\
20.0 & 2079 & 2639 & 2659 \\
40.0 & 726 & 1002 & 1047 \\
70.0 & 234 & 377 & 422 \\
100 & 38.6 & 115 & 157 \\
140 & 0.6 & 3.5 & 11 \\
\hline
\end{tabular}




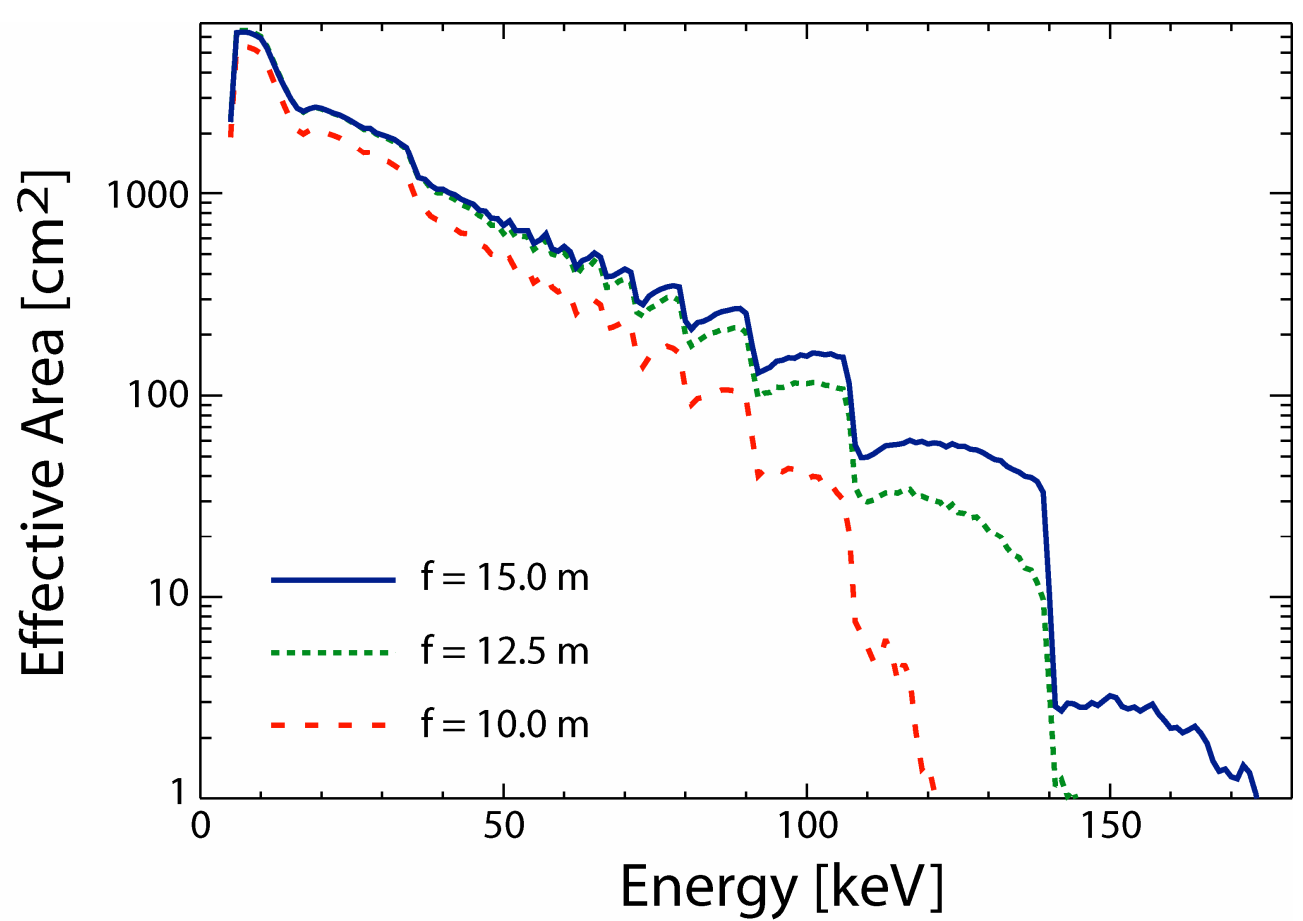

Figure 2. Total on-axis effective area versus energy for the NiV/SiC alone multilayer design for all three focal lengths.

The $15.0 \mathrm{~m}$ focal design results in a dramatic increase in effective area at higher energies due to the physical constraint on the minimum radius. Recall that the minimum radius is fixed at $5.9 \mathrm{~cm}$ for all focal length options to facilitate the fabrication of the telescope. As the focal length is increased, the graze angle of the inner-most shell decreases, providing more effective area at the highest energies. The smaller spread in effective area at lower energies results in the careful arrangement of modules per satellite and the total number of satellites, which tries to balance the total aperture area. For example, the percentage of full aperture used is $70 \pm 1 \%$ for all three focal length designs.

Figure 3 shows the effective area versus energy for several off-axis positions for the $f=15.0 \mathrm{~m}$ design. The decrement slowly increases as the energy increases: at $10 \mathrm{keV}$, the area at 5 ' off-axis is $43 \%$ that on-axis, while the ratio decreases to $15 \%$ at $100 \mathrm{keV}$. 


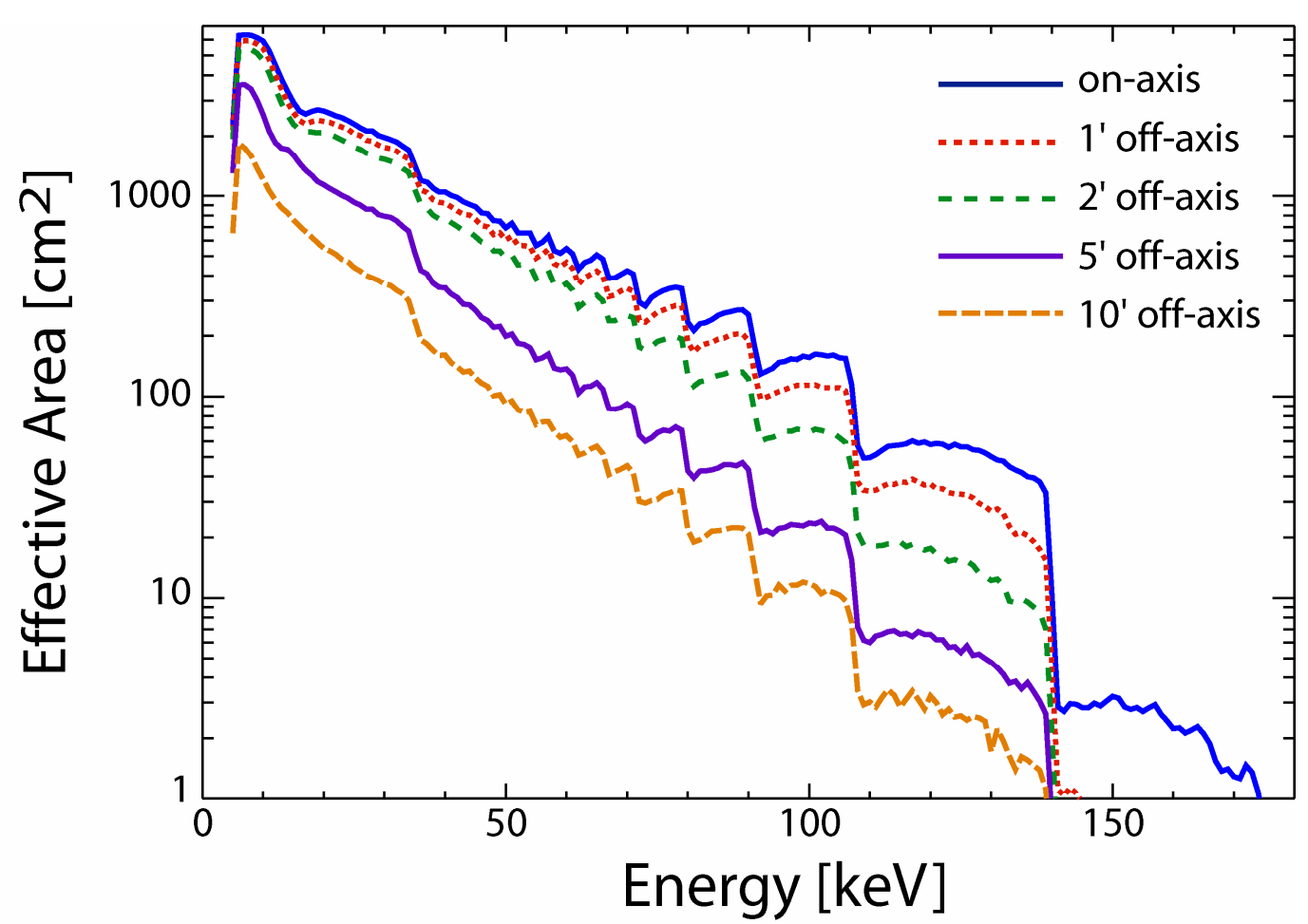

Figure 3. Effective area versus energy as a function of off-axis position for the baseline design at $\mathrm{f}=15.0 \mathrm{~m}$.

\subsubsection{NiV/SiC $+\mathrm{W} / \mathrm{SiC}$ multilayers}

Figure 4 compares the on-axis areas obtained from a multilayer design using NiV/SiC pairs on the top of the stack and $\mathrm{W} / \mathrm{SiC}$ pairs in the bottom of the stack to the pure $\mathrm{NiV} / \mathrm{SiC}$ design discussed in Section 4.1.1. This combination design outperforms the $\mathrm{NiV} / \mathrm{SiC}$ at all energies, with large gains achieved above $\sim 100 \mathrm{keV}$. Table 8 lists the on-axis area for the combination multilayer design at the same energies given in Table 7 and the subsequent gain (expressed as a ratio of areas). Note that the drop at $70 \mathrm{keV}$ is due to the deliberate choice of using only W/SiC for mirror groups 9-11 which leaves a residual effect on the overall effective area due the k-absorption edge of W. This could be smoothed out completely if we had chosen to use both material combinations for all mirror groups but we chose to boost the throughput slightly below $70 \mathrm{keV}$ for this specific design at the expense of leaving a small drop at $70 \mathrm{keV}$. This shows the variation and possibilities available with these new design options. 


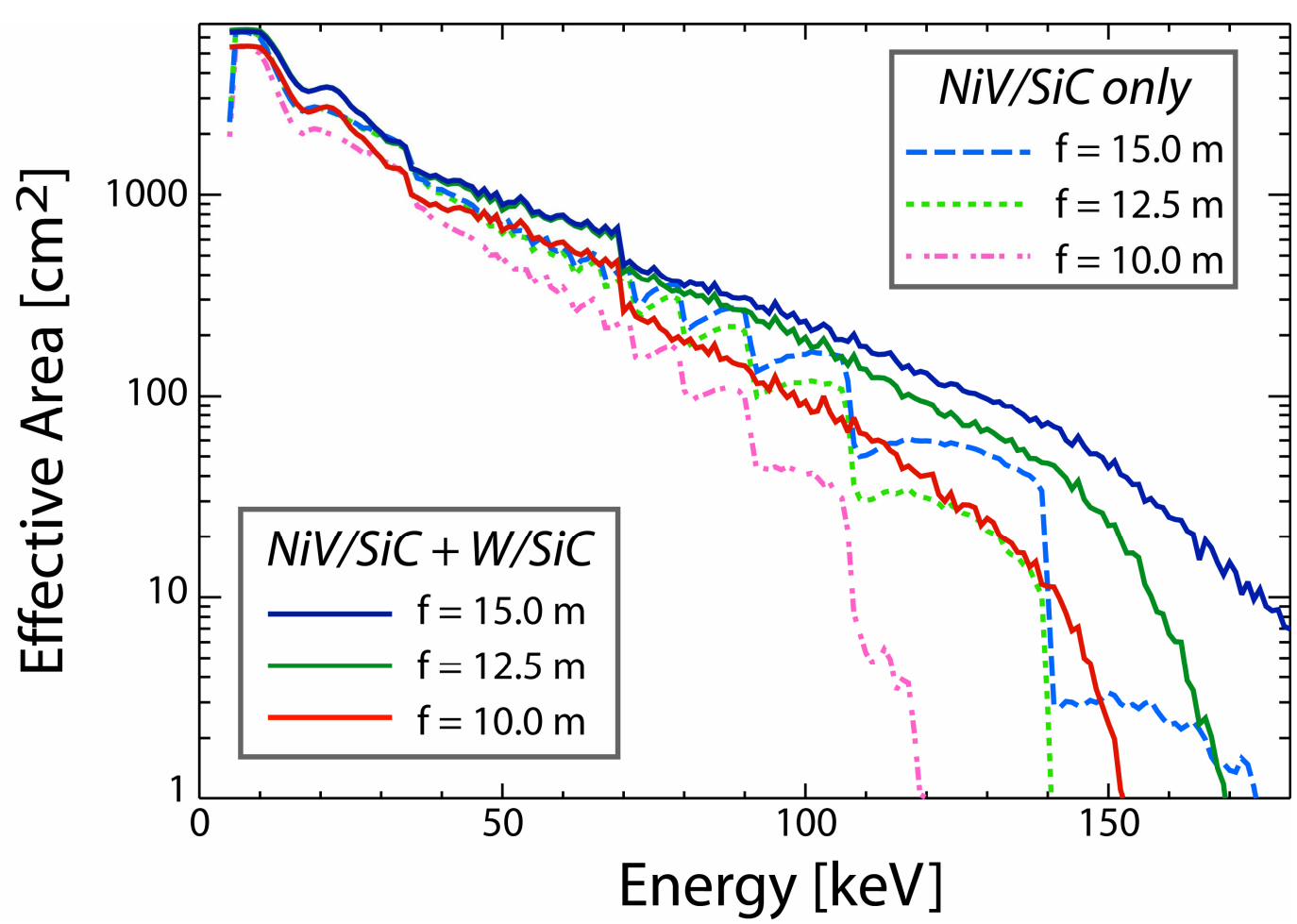

Figure 4. Comparison of the $\mathrm{NiV} / \mathrm{SiC}$ alone design to the combination multilayer layer design for all three focal lengths

Table 8: On-axis effective energy for the $\mathrm{NiV} / \mathrm{SiC}+\mathrm{W} / \mathrm{SiC}$ multilayer design and the gain over the $\mathrm{NiV} / \mathrm{SiC}$ design

\begin{tabular}{ccccccc}
\hline \hline & \multicolumn{3}{c}{ Effective Area $\left[\mathrm{cm}^{2}\right]$} & \multicolumn{3}{c}{ Gain $\left(\right.$ Area $_{\left.\mathrm{NiV} / \mathrm{SiC}+\mathrm{W} / \mathrm{Sic}: \text { Area }_{\mathrm{NiV} / \mathrm{SiC}}\right)}$} \\
Energy $[\mathrm{keV}]$ & $\mathrm{f}=10.0 \mathrm{~m}$ & $\mathrm{f}=12.5 \mathrm{~m}$ & $\mathrm{f}=15.0 \mathrm{~m}$ & $\mathrm{f}=10.0 \mathrm{~m}$ & $\mathrm{f}=12.5 \mathrm{~m}$ & $\mathrm{f}=15.0 \mathrm{~m}$ \\
\hline 10.0 & 5358 & 6478 & 6344 & 1.07 & 1.08 & 1.08 \\
20.0 & 2688 & 3376 & 3369 & 1.29 & 1.28 & 1.27 \\
40.0 & 859 & 1166 & 1198 & 1.18 & 1.16 & 1.14 \\
70.0 & 262 & 413 & 447 & 1.12 & 1.07 & 1.04 \\
100 & 94 & 195 & 235 & 2.44 & 1.70 & 1.50 \\
140 & 11 & 46 & 73 & 18.3 & 13.4 & 6.64 \\
\hline
\end{tabular}

\subsection{Sensitivities}

The sensitivities have been calculated assuming a $\Delta \mathrm{E} / \mathrm{E}$ of 0.5 , a signal to noise ratio of 3 , an Observation time of $10^{6}$ seconds, a background of $10^{-4} \mathrm{ph} / \mathrm{cm}^{2} / \mathrm{sec} / \mathrm{keV}$ and an HPD of 20 arcsec. Figure 5 gives the sensitivities for the NiV/SiC alone design and figure 6 gives the sensitivities for the combination material design and Figure 7 compares the sensitivity for the $15 \mathrm{~m}$ focal length case for the $\mathrm{NiV} / \mathrm{SiC}$ alone design and the combination design. Obviously signicant sensitivity is achieved up to and above $100 \mathrm{keV}$ with the combination material design outperforming the NiV/SiC alone 


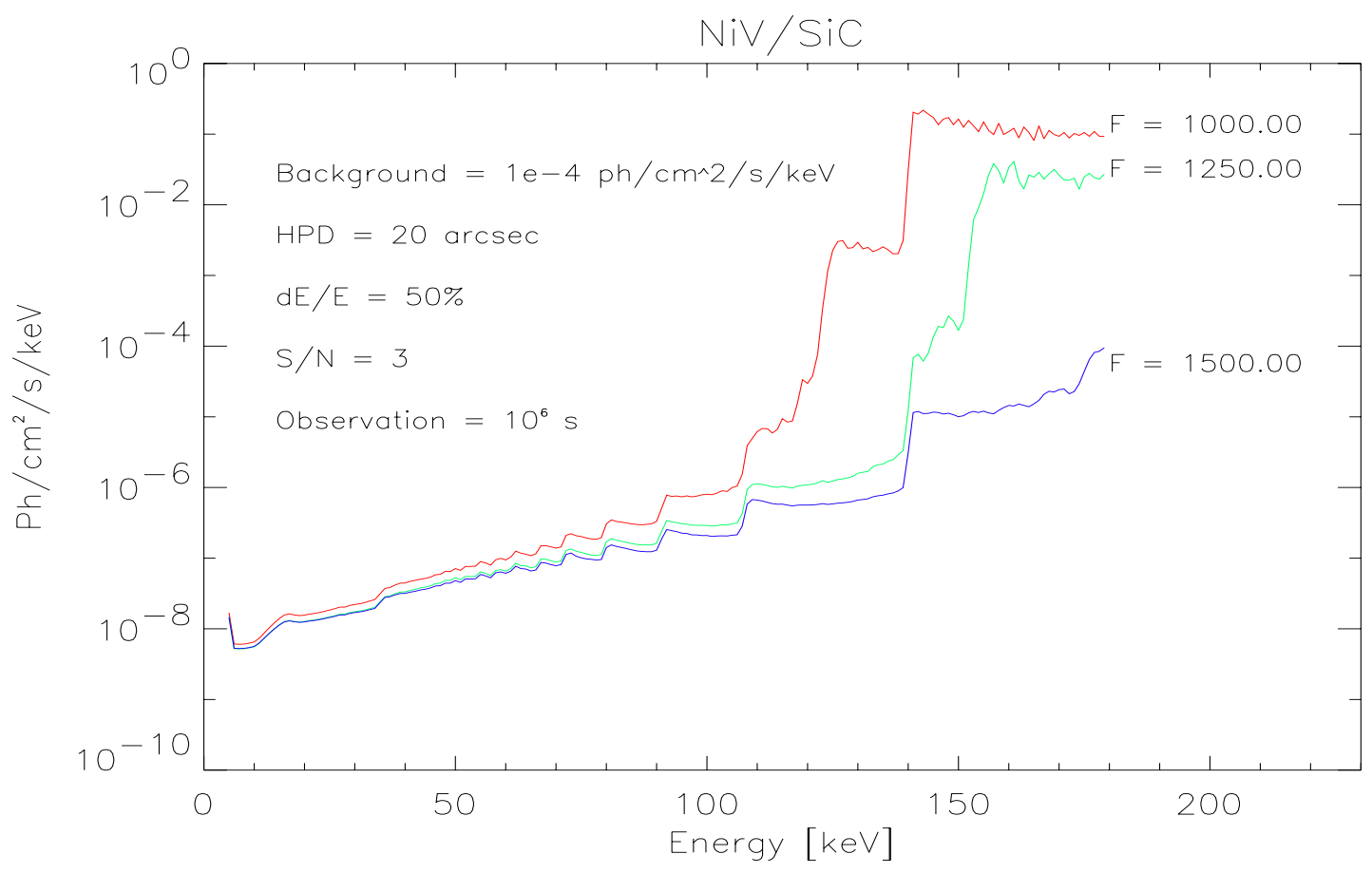

.Figure 5. The sensitivy for the $\mathrm{NiV} / \mathrm{SiC}$ alone design for the three focal length cases

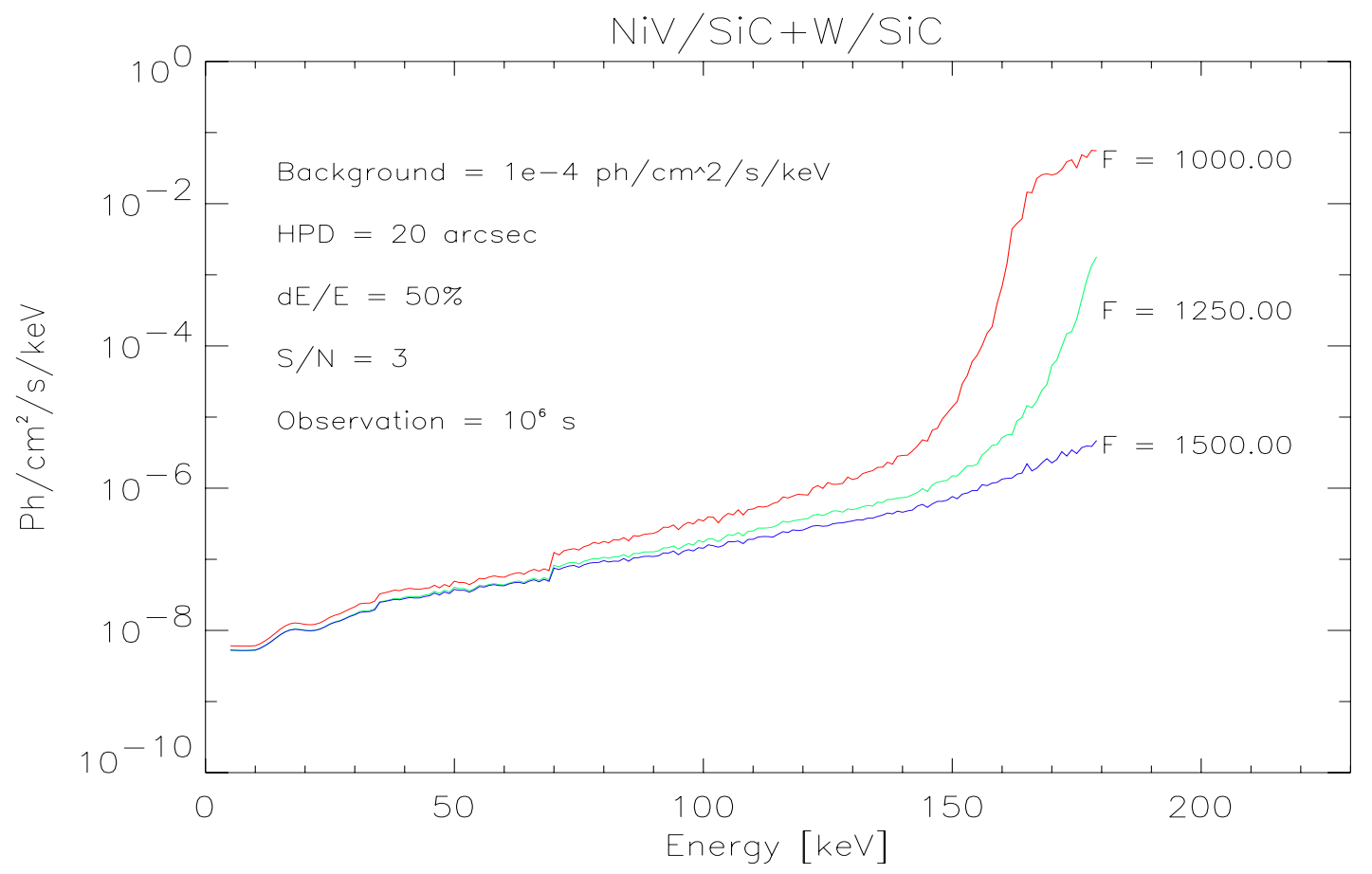

Figure 6 . The sensitivity for the combination material design for the three focal length cases 


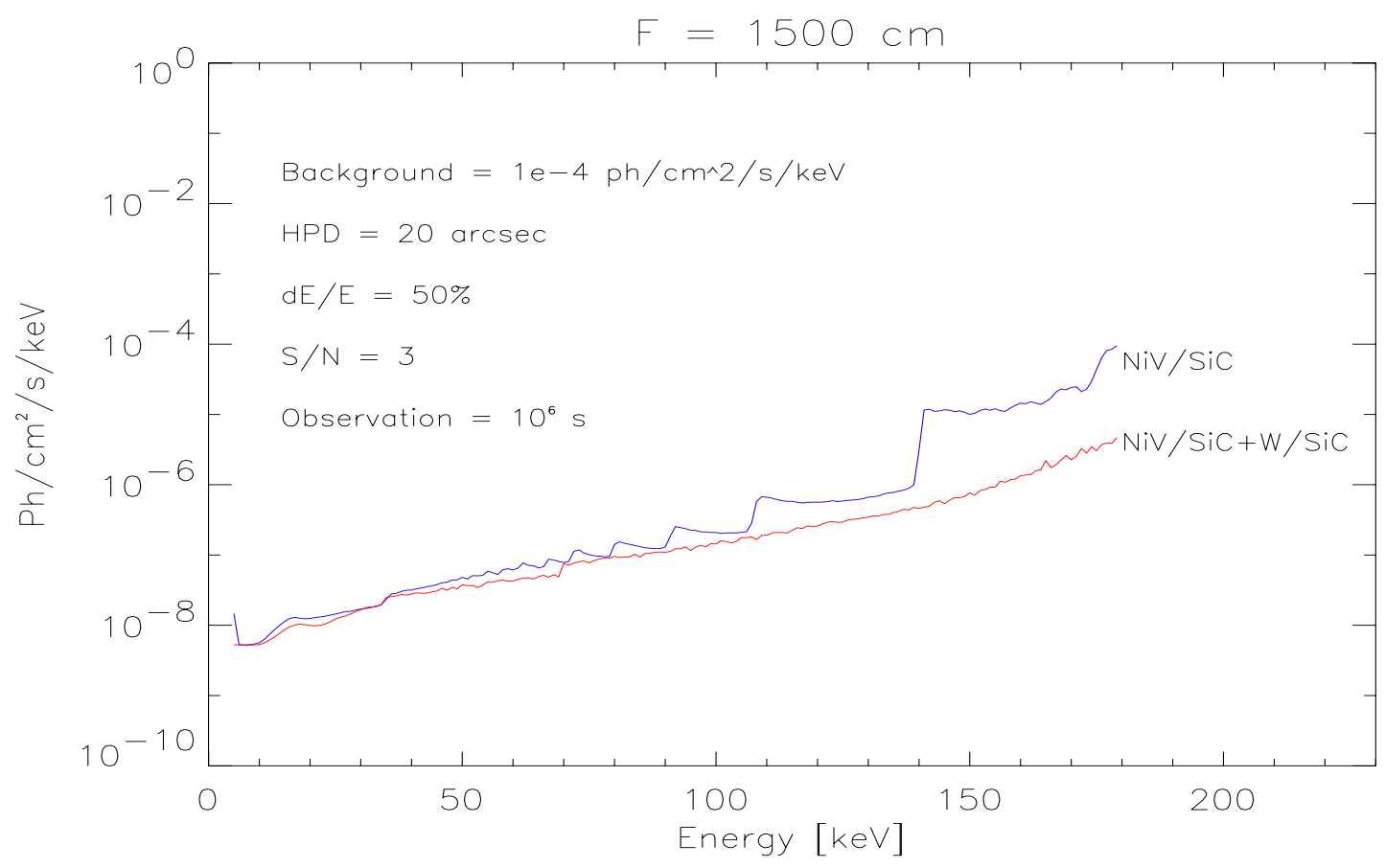

Figure 7. The Sensitivity for the $15 \mathrm{~m}$ focal length case for the NiV/SiC alone design and the combination design

\section{CONCLUSIONS}

We have shown that based on novel demonstrated multilayer coating capabilities it is possible to develop new hard Xray telescope designs for future high energy astrophysics missions which is not limited by sharp drops at k-absorption edges of the reflecting layers of graded multilayer coatings. In the specific example of the HXT on Con-.X this enables throughput up to and well above $100 \mathrm{keV}$ for reasonable focal lengths in the range between $10-15 \mathrm{~m}$. Specifically this new development speaks strongly in favor of the $15 \mathrm{~m}$ focal length case as significant throughput and sensitivity will be possible up to $150 \mathrm{keV}$. In addition It is worth noting that the design for the $\mathrm{NiV} / \mathrm{SiC}$ alone case is conservative and based on the Currently attainable minimum d-spacing of $35 \AA$ and a roughness of $5.5 \AA$. The enhanced NiV/C as developed by XENOCS holds promise for lower minimum d-spacing( as low as 20-25 $\AA$ ) and a smaller roughness of $3.5 \AA[6]$. We will follow this development closely and refine the Ni alone designs further in the future and compare to what can be achieved with the type of combination designs shown here.

\section{REFERENCES}

1. F.A.Harrison, S.Boggs, A.Bolotnikov, W.Cook, P.Mao, S.Schindler, C.Hailey, W.W.Craig, M.Jimenez-Garate, D.Windt, F.E.Christensen, C.P.Jensen, "Development of the balloon borne high energy focusing telescope mission", Proc. SPIE, Vol 4012, 693, 2000.

2. Y.Ogasaka, J.Tueller, K.Yamashita, P.Serlemitsos, R.Shibata, K.Tamura, A.Furuzawa, T.Miyazawa, R.Takahashi, M.Sakashita, K.Shimoda, Y.Tawara, H,kunieda, T.Okajima, H.Krimm, S.Barthelmy, Y.Soong, K.W.Chan, S.Owens, M.Rex, E.Chapin, M.Devlin, "First flight of a hard X-ray imaging experiment: The InFocus balloon flight", Proc. SPIE, Vol 5900, 5900-0R, 2005.

3. F.A.Harrison, "The Nuclear Spectroscopic Telescope Array (NuSTAR) Mission," Proc SPIE Vol 6266, These proceedings. 
4. N.E.White, H.D.Tannanbaum, A.E.Hornschemeier, M.R.Garcia, R.Petre, J.A.Bookbinder, "Constellation-X mission and implementation: Approach and status," Proc. SPIE, Vol 6266, These proceedings.

5. H.Kunieda, "New X-ray Telescope mission (NeXT): Current status of mission study", Proc SPIE, Vol 6266, These proceedings.

6. D.H.Lumb ,M.Bavdaz, F.E.Christensen, A.Dariel, P.Høghøj, M.K Krumrey, C.P.Jensen, K.K.Madsen, E.Zeigler, "Multilayer coating development for XEUS," Proc. SPIE, Vol 6266, These proceedings.

7. J.Knoedlseder on behalf of the GRI consortium, "GRI: The Gamma-Ray Imager mission," Proc SPIE, Vol 6266, These proceedings.

8. R.Diehl,F.X.Timmes,"Gamma-Ray line emission from radioactive isotopes in stars and galaxies," PASP, Vol 110, 637,1998 .

9. F.A.Harrison, S.E.Boggs, F.E.Christensen, N.A.Gehrels,J.E.Grindlay, C.H.Chen, W.W.Craig, C.J.Hailey, P.Pnto, S.E.Thorsett, J.Tueller, D.L.Windt, S.E.Woosley, "High Resolution spectroscopic Imaging Mission," Proc. SPIE, Vol 4851, 345, 2002.

10. C.P.Jensen, K.K.Madsen, F.E.Christensen, "Investigation of new material combinations for hard X-ray telescope designs," Proc. SPIE, Vol 6266, These proceedings.

11. C.P.Jensen, K.K.Madsen, F.E.Christensen, "W/SiC and Pt/SiC multilayers for the NuSTAR hard X-ray telescope," Proc. SPIE, Vol 5900, 5900-07, 2005.

12. D.L.Windt, S.Donguy, C.J.Hailey, J.E.Koglin, E.Ziegler, F.E.Christensen, F.A.Harrison, "Optical constants for hard X-ray multilayers over the energy range E $=35 \mathrm{keV}-180 \mathrm{keV}$," Proc. SPIE, Vol 5168, 35, 2003.

13. W.W.Zhang, D.A.Content, J.P.Lehan, R.Petre, T.T.Saha, M.Gubarev, W.D.Jones, S.L.O'Dell, "Development of lightweight X-ray mirrors for the Constellation-X mission,” Proc. SPIE, Vol 5900, 5900-0V, 2005.

14. P.Mao, F.A.Harrison, D.Windt, F.E.Christensen, "Optimization of graded multilayer designs for astronomical X-ray telescopes," App Opt, Vol 38, 4766, 1999. 